\title{
A Prototype Sourceless Kinematic-Feedback Based Video Game for Movement Based Exercise.
}

\author{
J.Foody, D.Kelly, D.Kumar, D.Fitzgerald, T.Ward, B.Caulfield, C.Markham
}

\begin{abstract}
This paper presents a prototype kinematic and audio feedback based video game, availing of a scalable motion capture acquisition system, based around a number of orientation sensors. The orientation sensors used are USB based tri-axis magnetic and gravitational field transducers. The novel video-game is capable of incorporating the real time data from these sensors to control an on screen avatar, which in turn can be programmed to give appropriate instructions to the user i.e. play a sound file, once the user obtains a certain posture. The video game is designed to promote physical exercise and movement based relaxation, in particular; Yoga. In addition, design considerations; implementation and performance of the system are analyzed, discussed and the accuracy qualitatively analyzed by comparing movement data obtained from it to that of a validated motion analysis technique, the CODA motion analysis system.
\end{abstract}

\section{INTRODUCTION}

$\mathrm{I}^{\mathrm{N}}$ this project we are developing an effective feedback system for a human interface to promote mental and physical exercise and relaxation. Therapies such as Yoga and Tai Chi have been shown to have many therapeutic benefits in rehabilitation including enhancing postural awareness and reducing chronic pain and hypertension. We are designing a garment based physiological and kinematic measurement system in order to investigate these therapies. A system overview of which is shown in figure 1. The system is to be integrated into a video game. The garment is the human interface to a real-time kinematic feedback based video game, for promotion of yoga and Tai Chi.

Manuscript received April 3, 2006. This work was financed by the Higher Education Authority of Ireland.

J. Foody is with the Computer Science Department at the National University Maynooth Ireland, Eire (phone: 353-1-70803847; fax: 353-1708 3848; e-mail: jfoody@ cs.nuim.ie).

D. Kelly is with the Computer Science Department at the National University Maynooth Ireland, Eire.

D. Kumar, D. Fitzgerald is with School of Physiotherapy and Performance Science University College Dublin, Eire.

Dr. T. Ward is with the Department of Electronic Engineering, National University of Ireland Maynooth, Eire.

Dr. B. Caulfield is with School of Physiotherapy and Performance Science University College Dublin, Eire.

Dr. Charles Markham is with the Computer Science Department, National University of Ireland Maynooth, Maynooth, Co. Kildare, Eire.

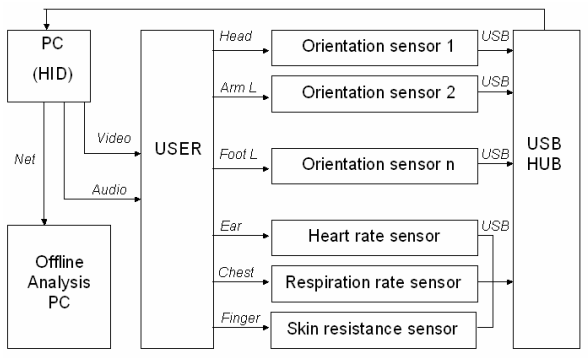

Figure 1: Proposed System Overview.

In order to achieve this, the development of an orientation sensor capable of scalable deployment was essential, multiples of which could be embedded on clothing worn by the subject at key kinematic tracking points on the body.

The sensor, an integrated tri-axis magnetic and inertial sensor package has been designed and implemented [1]; it is a Universal Serial Bus (USB) kinematic transducer, and therefore capable of scalable deployment. Moreover it is unobtrusive and because of its utilization of SMD technology has the ability to be miniaturized and realized on a small footprint. The orientation sensor developed is shown in figure 2 below:

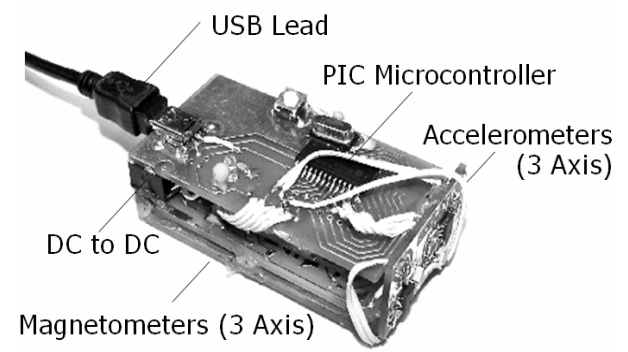

Figure 2: Prototype, USB Based Orientation Sensor.

In our specific application a minimum of nine orientation sensors are needed for full human motion tracking. A way to access data from anyone of these sensors at any time had to be realised. A software system to manage the introduction of multiple sensors was developed [2].

The sensor(s) are connected to the Host via a USB hub. Using a pre-compiled Dynamic Link Library (DLL), 
developed in the Visual Studio $6.0 \mathrm{C}++$, programming environment; a function call provides the user or software developer, with real-time access to kinematic data from any one of the connected sensors. The function call can be used in a series of programming environments, including the .NET framework.

This system has been integrated into a novel kinematic feedback based video - game for the study of movement based exercise, in particular Yoga. The video-game provides the user with a visual representation of their movements via an on screen avatar; in addition, it is capable of providing instructions or audio feedback to the user i.e. informing the user whether or not they have obtained a specified posture correctly.

Further features include, a movement record function, a novel playback functionality which allows synchronization of sound files during motion playback, the ability to render any skeletal shape; human or non human, a zoom and pan feature, and finally an open source API to allow further development of features in the game.

A picture of the avatar instructor tracking arm motion of a user can be observed in figure. 3

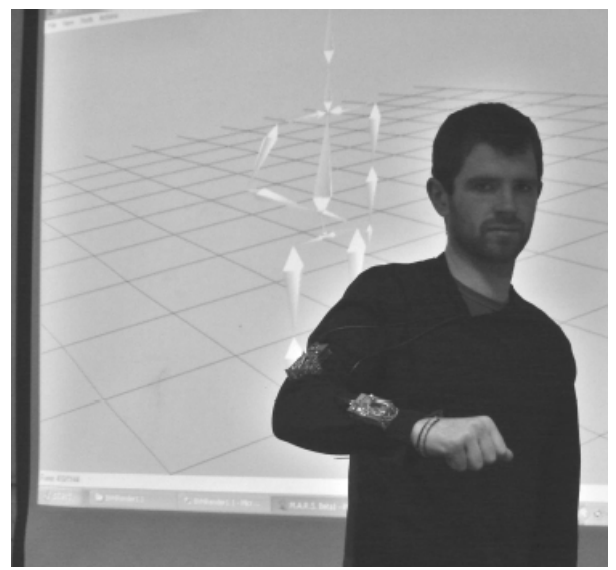

Figure 3: Integration of prototype orientation sensors with the Video Game.

Finally to assert how accurate these new sensors are, they were compared with a standard method of measurement. For this testing procedure we used a movement analysis system known as the CODA Motion Analysis System (Charnwood Dynamics Ltd., Victoria Mills, Fowke Street, Rothley, and Leicestershire, LE7 7PJ, United Kingdom).

The CODA analysis system is a validated method of assessing body movements and comprises of three optical sensors mounted to a rigid frame in a scanner unit. The scanner is used to capture infrared light signals pulsed sequentially by markers placed on anatomical landmarks. The middle sensor captures vertical movements of the markers and the outer sensors capture the horizontal movements. This system is commonly used in clinical research with a capacity to measure many variables including 3D position, velocity and acceleration of markers to yield data such as segmental rotation, joint moments, joint power and angular displacements. However, experiments carried out in this study only required angular displacement of the knee flexion-extension movement.

\section{Materials an Methods}

The rendering of the video games avatar is based on a motion capture file format called BVH (BioVision Hierarchical data). This file format provides a header section which defines the translation data for the hierarchical and initial pose of the avatar in the form of a tree structure [3] and a data section which consists of the number of frames in an animation sequence, the length of time for each to occur and the rotational data or Euler Angles of rotation for each node/body part tracked in each frame. Meaning for full body motion capture the global representation of any particular node is controlled by the accumulative movement of all nodes between it and the root, which we chose to be the hip due to its limited movement.

This video game is designed in a modular fashion, split in to a number of components based on functionality. These include a BVH parsing component, motion and skeleton/avatar data structures, a sound playback component, a graphics rendering component and a graphical user interface (GUI) allowing user interaction with the different functions of the software.

Within the graphics rendering and GUI components, certain methods are made publicly available to allow a programmable interface for users who wish to animate motion data from currently unsupported file formats and devices in the future.

The game is programmed in C Sharp and the .Net framework libraries are utilized for the GUI components of the system, in addition DirectX 9 libraries are used for the 3D graphics rendering components of the game. Functionality provided by the .Net framework includes predefined windows graphical components. DirectX provides the interface to the local machines graphics hardware as well as data types and functions to store and animate 3D objects on screen. The graphics processing of the skeletal rendering and animation is performed on the local computers graphics/video card, and the system assumes the presence of a DirectX compatible video or graphics card.

In order to achieve real time kinematic feedback the game was integrated with a system which provided access to any and all motion capture sensors connected to the host P.C by use of a pre-programmed DLL, imported into the project by use of the .NET DLLimport property.

Using this, sensors connected to the USB host were polled every 40 milliseconds, the real time data returned to the game from each sensor. This meant that 3 Euler angles of rotation could then be assigned to move a particular node/body segment e.g. the forearm of the avatar in the 
game. This live animation of the tracked abides by the same rules which are used for the playback of BVH motion data; therefore, if a single bone is animated on screen, then all descendants of this bone (i.e. children, grandchildren etc.) will follow the animation of its parent. A runtime display of the application can be seen in figure 4 .

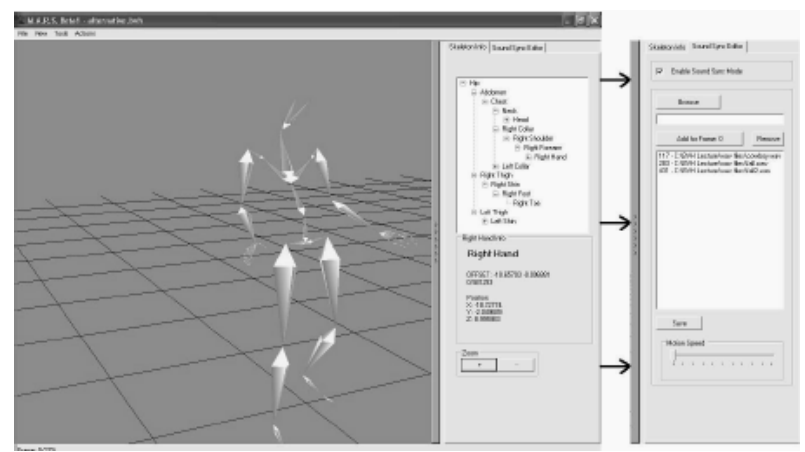

Figure 4: Runtime Display of Application.

\section{Experiments and Results}

The motion capture sensor this game is based on has been commissioned [1]. On foot of this the basis for the videogame i.e. access to a multiple sensor system provided by a pre-compiled DLL, was tested. To do this one healthy subject was required for the study; the subject had no past history of neurological or musculoskeletal disease.

In acquiring knowledge of how accurately the sensors could measure 3-d movement of the body and by extrapolation how accurate the kinematic feedback in the game environment would prove, a double leg squat movement was chosen for the test procedure. This particular movement was chosen, as it is a functional body movement that is efficient to analyze and reproduce. The kinematic sensors used in the game were attached with one on the front of the thigh and one on the lateral border of the lower leg. CODA markers were placed on the greater trochanter, later femoral condyle, head of fibula and lateral malleolus in order to create vector coordinates for both upper and lower leg segments. The subject along with attached sensors can be viewed in Figure 5. From these coordinates we were able to derive knee angular displacement throughout the movement. Data was acquired using a sampling frequency of $25 \mathrm{~Hz}$

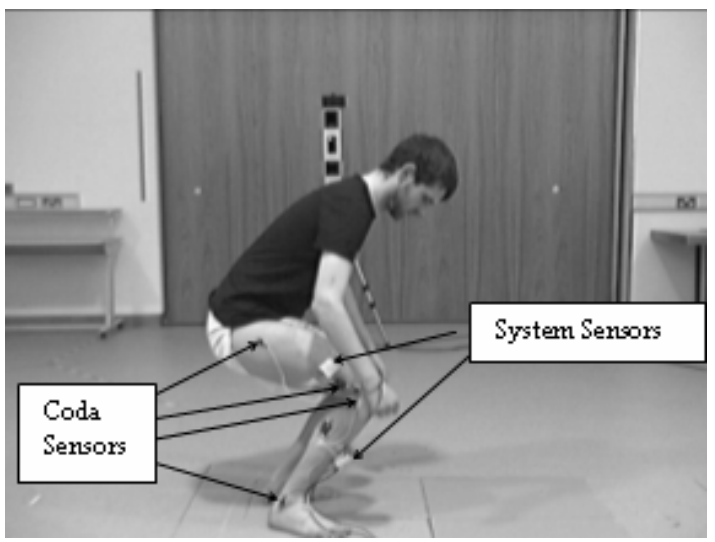

Figure 5: Squat experiment process.

A synchronized data collection then took place for one double leg squat movement over a seven second period. The results of which, the degree change between the two coda vectors and the angle of change of the pitch rotation between the two developed sensors were recorded and plotted. This can be observed in figure 6 , the dashed line representing the Coda Measurement and the continuous line representing the Sensor measurement.

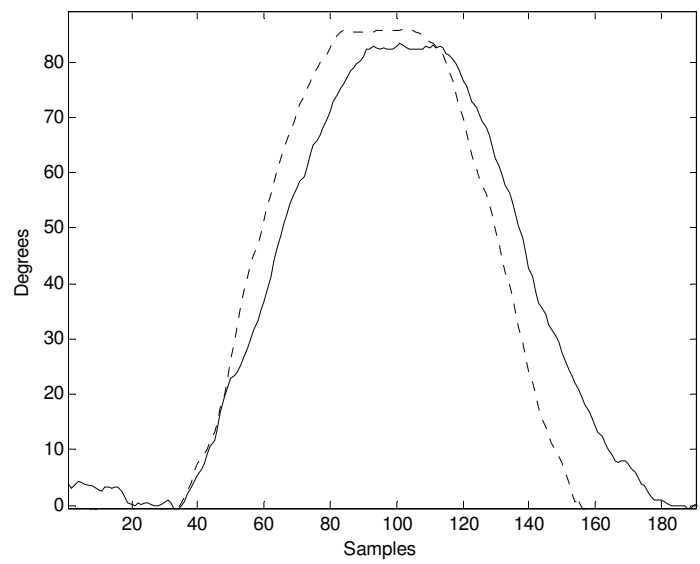

Figure 6: Coda versus Sensors for Double Leg Squat movement.

In addition to this a series of tests were performed on the video game itself. White Box testing, availing of the debugging tools provided within the .Net, such as break point operators and debug message writing were utilized. Black Box testing provided for the use of a number of different test cases for the game. They included functional, usability, fault component integration test cases. 


\section{Discussion}

The experiment results show that the developed syatem and CODA provide similar response under the same tilt conditions, the notable difference being a lag in the tracking of the double squat movement. This can be attributed to one main factor, the running averaging performed on all three axes of both the Magnetic field and gravitational field vectors over twenty values in the DLL utilized by the game, before the Euler angles are found from them. This is done to reduce noise in the sensors output.

However, this lag as can be observed in figure 6 to average approximately ten samples, therefore as the two sensors being polled simultaneously can perform at rates up to 25 samples per second; the lag amounts to an average of less than half a second delay. This delay can be reduced further by an upgrade to a USB 2.0 interface.

As the video game accepts these Euler angles and replicates them in the form of movement of an on screen avatar, it can be said that the video-game can track a double-squat movement and by extension numerous additional hinge joint movements performed by the human body e.g. an arm.

Furthermore, at the moment our system is wired, i.e. requires a wire from the USB hub on the body, which the sensors connect to, for both power and data transfer. It is however, worth noting that once the backward compatibility of the forthcoming wireless USB standard is utilized, a wireless human motion capture sensor and system can be achieved with ease, considering there will be no loss in data rates, and a battery pack is used [4].

\section{Conclusion}

This paper describes the development of a software system to dynamically render and animate a skeleton using data acquired from a motion capture data source, i.e. a DLL based kinematic data acquisition system developed from multiple USB based inertial and magnetic field transducers. Any given skeleton, human or non human, can be rendered and animated using motion data, acquired from afore mentioned system. The software was developed as part of a project which aims to help people learn the skill of yoga via a Yoga Instructor video game i.e. a kinematic, audio and eventually bio-feedback video-game based environment.

The DLL kinematic data acquisition system which the video-game is based on and therefore its abilities inextricably linked to that of the video-game, has been proven to track human movement of a hinge joint to within a less than half a second of that movement occurring, in a comparison study against the standardized CODA system. Future work will include a full commissioning of the videogame through a comprehensive user study, involving multiple yoga performers with varying levels of ability.

In conclusion a prototype Yoga Instructor game, based on kinematic and audio feedback has been developed.

\section{ACKNOWLEDGMENTS}

The authors would like to thank the Higher Education Authority of Ireland for their support in funding this project and those involved at the National University of Ireland Maynooth and University College Dublin.

\section{REFERENCES}

[1] J.Foody D.Kumar D.Fitzgerald B.Caulfield T.Ward C.Markham, "A USB Interfaced Motion Capture Sensor, Using Tri-Axis Magnetic/Inertial Sensors For Use In Kinematic Studies.” IEEE European conference on Biomedical Engineering. - Embec, Prague. November 2005.

.[2] John Foody, Daniel Kelly, Damini Kumar, Diarmaid Fitzgerald, Brian Caulfield, Tomas Ward and Charles Markham, "A Real Time Motion Capture System, Using USB Based Tri-Axis Magnetic and Inertial Sensors, for Movement Based Relaxation" IEE Irish Signals and Systems Conference,2006, - D.I.T, Dublin - June 2006

[3] Herda, P.Fua, R.Plankers, R.Boulic and D.Thalmann, "SkeletonBased Motion Capture for Robust Reconstruction of Human Motion" - CH-1015 Lausanne, Switzerland

[4] Wireless USB, A White Paper http://www.intel.com/technology/comms/wusbdownload/ Accessed: 11/09/05 